\title{
Structural Landscapes in Geometrically Frustrated Smectics
}

\author{
Jingmin Xia $\odot,{ }^{1}$ Scott MacLachlan $\odot,{ }^{2}$ Timothy J. Atherton, ${ }^{3, *}$ and Patrick E. Farrell ${ }^{1, \dagger}$ \\ ${ }^{1}$ Mathematical Institute, University of Oxford, Oxford OX2 6GG, United Kingdom \\ ${ }^{2}$ Department of Mathematics and Statistics, Memorial University of Newfoundland, St. John's, Newfoundland A1C 5S7, Canada \\ ${ }^{3}$ Department of Physics and Astronomy, Tufts University, Medford, Massachusetts 02155, USA
}

(Received 21 January 2021; accepted 31 March 2021; published 27 April 2021)

\begin{abstract}
A phenomenological free energy model is proposed to describe the behavior of smectic liquid crystals, an intermediate phase that exhibits orientational order and layering at the molecular scale. Advantageous properties render the functional amenable to numerical simulation. The model is applied to a number of scenarios involving geometric frustration, leading to emergent structures such as focal conic domains and oily streaks and enabling detailed elucidation of the very rich energy landscapes that arise in these problems.
\end{abstract}

DOI: 10.1103/PhysRevLett.126.177801

Smectic liquid crystals are complex fluids that exhibit orientational order and a layered structure over macroscopic distances [1]. Since the layers are nearly incompressible, an immediate consequence is that the material prefers to locally adopt one of six families of surfaces (in three dimensions) compatible with constant layer spacing [2-5]. External constraints may force deformations of the smectic that are incompatible with the layer constraint, leading to geometric frustrations and the spontaneous assembly of a wide variety of textures with characteristic defect structures of the smectic phase $[4,6]$. Driven by advances in surface control, there has been considerable renewed interest in exploiting the ability of smectics to repeatably self-assemble over device length scales by using surface patterning $[7,8]$, topographical features such as grooves [9-12] or posts [13,14], confinement in droplets [15-17], or curved surfaces more generally [18], to produce emergent patterns $[19,20]$ that are optically active as lenses, gratings [21], photonic crystals [20], or lithographic templates [22]. Moreover, defect structures in the texture act to efficiently trap dispersed microparticles or nanoparticles, making smectics useful for hierarchical [23-25] or synergistic [26] assembly processes that could potentially be adopted for metamaterial, sensor, or solar cell production. Since many of the remarkable properties of smectics arise because of the geometric and topological consequences of layering, they form a paradigmatic model system to understand geometric frustration in other lamellar phases such as block copolymers [27,28], membranes, and vesicles [29,30].

The very complicated structures that emerge in frustrated smectics have, however, proven to be very challenging to model mathematically. While many of the observed textures have been understood through elegant geometric approaches $[3,4,13,18-20,26,31-35]$ or by perturbing from the nematic phase [36-38], to date there have been few successful efforts to use numerical methods to predict the structures adopted by smectics in general configurations. Such methods could be of great benefit to structure prediction where the defects cannot be observed optically, for example, in thin films [39-43]. Furthermore, scenarios where partial smectic order exists, such as during the transition from the nematic to the smectic phase, may exhibit very complicated pretransitional structures $[37,38,44,45]$, and few studies have addressed the connection between pattern formation and the peculiar critical behavior of liquid crystals at the nematicsmectic transition [46]. Dynamical phenomena-such as time-varying layer spacing [47], interactions between embedded particles [31], and the evolution of smectic films and bubbles [48-50] —also present difficult problems that appear to require numerical modeling.

One major obstacle to successful modeling of smectics is the complicated nature of the smectic order. In the original theory of de Gennes [1], the smectic phase is characterized by a complex order parameter $\psi(\mathbf{r})=|\psi(\mathbf{r})| e^{i \phi(\mathbf{r})}$ that contains both the amplitude and phase of the density modulations. It is a remarkably successful approach, providing a theory of the nematic-smectic-A transition analogous to the Ginzburg-Landau theory of superconductivity. Nonetheless, it presents certain challenges, as reviewed in Pevnyi et al. [51]. The first issue is due to the topology of the complex order parameter $\psi$ itself: $\operatorname{Im}(\psi)$ does not contain physical information. Second, this model is formed on a coarse-grained basis; i.e., this energy does not represent the local free energy density on the length scale of the smectic layers themselves. To amend these issues, Pevnyi et al. propose a theory formulated in terms of a real-valued variation $\delta \rho(\mathbf{r})$ from the average density and a director field $\mathbf{n}(\mathbf{r})$, the local axis of average molecular alignment. Using a real-valued density variation avoids many of the problems of alternative approaches such as using double-valued complex order parameters [51]. Nonetheless, this theory, as presented, is not able to 
reproduce half-charge defects because of the presence of director discontinuities in these defects [52], which cannot be characterized by a continuous vector field. For example, around a $\pm 1 / 2$ defect where $\mathbf{n}$ rotates by $\pm \pi$ degrees, a discontinuity line where $\mathbf{n}$ reverses sign must exist. In fact, since $\mathbf{n}$ enters the model only through the tensor $N=\mathbf{n} \otimes \mathbf{n}=\mathbf{n}_{i} \mathbf{n}_{j}$, Pevnyi et al. solve for $N$ in their implementation, which allows them to represent halfcharge defects [52], but numerically enforcing that $N$ is a line field (i.e., of the form $\mathbf{n} \otimes \mathbf{n}$ for some unit vector $\mathbf{n}$ ) in minimization is difficult [53].

In this Letter, we formulate a theory of smectics suitable for finite element simulation and apply it to several partially understood problems involving the configuration of smectics between antagonistic boundary conditions, i.e., those that favor opposing orientations incompatible with the layer constraint. We quantitatively study the transition from uniform layering to the formation of defects [54], examine the role of imposed surface orientation on the configuration of focal conic domains [44], and predict the structure of oily streaks that occur in very thin smectic films $[21,41,43]$.

We begin with Pevnyi et al.'s proposed energy [51],

$$
\begin{aligned}
F(\delta \rho, \mathbf{n})= & \int_{\Omega}\left[\frac{a}{2}(\delta \rho)^{2}+\frac{b}{3}(\delta \rho)^{3}+\frac{c}{4}(\delta \rho)^{4}\right. \\
& \left.+B\left|\mathcal{D}^{2} \delta \rho+q^{2} \mathbf{n} \otimes \mathbf{n} \delta \rho\right|^{2}+\frac{K}{2}|\nabla \mathbf{n}|^{2}\right],
\end{aligned}
$$

which is to be extremized to obtain stationary solutions $\delta \rho$ and $\mathbf{n}$ subject to the pointwise constraint $\mathbf{n} \cdot \mathbf{n}=1$. The first three terms in Eq. (1) with coefficients $a, b$, and $c$ are a Landau-de Gennes expansion of the free energy and set the preferred value of $\delta \rho$ in the uniform state; $q$ is the wave number of the layering, $B$ is a nematic-smectic coupling parameter, $\mathcal{D}^{2}$ denotes the Hessian operator, $K$ is the elastic constant, and $\Omega$ is the domain of integration. The functional (1) can be derived from density-functional theory (based on a molecular statistical description), analogous to earlier work on smectics $[55,56]$.

Noticing the fact that Eq. (1) depends only on elements of the dyad $\mathbf{n}_{i} \mathbf{n}_{j}$, Ball and Bedford [57] proposed to modify Eq. (1) by replacing $\mathbf{n}_{i} \mathbf{n}_{j}$ by a uniaxial representation $\left(Q / s+I_{d} / d\right)_{i j}$, leading to

$$
\begin{aligned}
F(\delta \rho, Q) & =\int_{\Omega}\left[\frac{a}{2}(\delta \rho)^{2}+\frac{b}{3}(\delta \rho)^{3}+\frac{c}{4}(\delta \rho)^{4}\right. \\
& \left.+B\left|\mathcal{D}^{2} \delta \rho+q^{2}\left(\frac{Q}{s}+\frac{I_{d}}{d}\right) \delta \rho\right|^{2}+\frac{K}{2}|\nabla Q|^{2}\right] .
\end{aligned}
$$

Here, $s$ is the scalar order parameter, $I_{d}(d \in\{2,3\})$ is the identity matrix, and $Q$ is a tensor-valued order parameter. There is no longer any constraint imposed on the state variables. They proved the existence of minimizers of their modified model but did not pursue any numerical analysis or realize any implementation. One can anticipate numerical difficulties caused by having $s$ on the denominator, as it is likely to be near zero for defect structures of physical interest.

Inspired by the modification from Ball \& Bedford [57], we propose the following alternative energy functional:

$$
\begin{aligned}
F(\delta \rho, Q)= & \int_{\Omega}\left[\frac{a}{2}(\delta \rho)^{2}+\frac{b}{3}(\delta \rho)^{3}+\frac{c}{4}(\delta \rho)^{4}\right. \\
& +B\left|\mathcal{D}^{2} \delta \rho+q^{2}\left(Q+\frac{I_{d}}{d}\right) \delta \rho\right|^{2} \\
& \left.+\frac{K}{2}|\nabla Q|^{2}+f_{n}(Q)\right],
\end{aligned}
$$

where the nematic bulk energy density $f_{n}(Q)$ is $-l\left(\operatorname{tr}\left(Q^{2}\right)\right)+l\left(\operatorname{tr}\left(Q^{2}\right)\right)^{2}$ in two dimensions and $-l / 2\left(\operatorname{tr}\left(Q^{2}\right)\right)-l / 3\left(\operatorname{tr}\left(Q^{3}\right)\right)+l / 2\left(\operatorname{tr}\left(Q^{2}\right)\right)^{2} \quad$ in three dimensions.

We pause to contrast Eq. (3) with Ball \& Bedford's formulation, Eq. (2). In order to avoid possible numerical issues caused when $s \approx 0$, we instead weakly enforce $s=1$ by adding the nematic bulk term $f_{n}$. The global minimizer of the nematic bulk energy $\int_{\Omega} f_{n}(Q)$ is known to be a uniaxial $Q$ tensor with scalar order parameter $s=1$ (see Proposition 15 in Ref. [58]). Thus, inclusion of this term promotes both the favorable scalar order parameter and a tendency towards a uniaxial expression for $Q$.

A substantial difficulty in obtaining the numerical solution of the minimization problem with Eq. (3) arises from the presence of the Hessian term, which requires $\delta \rho \in$ $\mathcal{H}^{2}$ (i.e., square-integrable functions with square-integrable first and second derivatives). A conforming discretization requires the use of $\mathcal{C}^{1}$-continuous elements (i.e., the approximation is continuous with continuous first derivatives). Constructing these finite elements is quite involved in practice, especially in the three-dimensional case. Therefore, we use nonconforming discretizations following the so-called $\mathcal{C}^{0}$ interior penalty approach [59]. Essentially, we use $\mathcal{C}^{0}$-conforming elements (i.e., continuous without necessarily continuous first derivatives) and penalize interelement jumps in the first derivatives to weakly enforce $\mathcal{C}^{1}$ conformity. To this end, we add a penalty term to the energy functional (3), leading to

$$
F_{\gamma}(\delta \rho, Q):=F(\delta \rho, Q)+\sum_{e \in \mathcal{E}_{l}} \int_{e} \frac{\gamma}{2 h_{e}^{3}}(\llbracket \nabla \delta \rho \rrbracket)^{2} .
$$

Here, $\gamma$ is the penalty parameter (we fix $\gamma=1$ throughout this work), $\mathcal{E}_{I}$ is the set of interior facets (edges/faces) of a mesh, $h_{e}$ denotes the size of an edge/face $e$, and the jump operator of a vector $\nabla w$ on a facet $e$ of two adjacent cells, labeled $K_{-}$and $K_{+}$, is defined as $\llbracket \nabla w \rrbracket=(\nabla w)_{-} \cdot \nu_{-}+(\nabla w)_{+} \cdot \nu_{+}$, with $\nu_{-}$and $\nu_{+}$denoting the restriction of the outward normal to 
$K_{-}$and $K_{+}$, respectively. The numerical analysis of this discretization will be reported elsewhere. Using a $\mathcal{C}^{0}$ interior penalty method has the advantages of both convenience and efficiency: The weak form is simple, with only minor modifications from a conforming method, and fewer degrees of freedom are used than with a fully discontinuous method.

We now apply our discretization of Eq. (3) to a class of problems that encompasses commonly used techniques to induce self-organized structures in smectics. The liquid crystal is confined between two substrates treated to promote different preferred molecular orientations and must somehow interpolate between them, but unlike a nematic liquid crystal that can achieve this smoothly, a smectic may be prevented from doing so because of the layer constraint.

As a simple example, proposed in the classic work of Williams and Kléman [54], consider the situation depicted in Fig. 1, where we impose the director $\mathbf{n}_{e}=$ $\left(\cos \theta_{0},-\sin \theta_{0}\right)$ for fixed $\theta_{0} \in[0, \pi / 2]$ at the lower boundary and $\mathbf{n}_{e}=\left(\cos \theta_{0}, \sin \theta_{0}\right)$ at the upper boundary. The corresponding boundary data for the $Q$ tensor derived from $\mathbf{n}_{e}$ are given in the Supplemental Material [60]. For $\theta_{0}=0$, the boundary conditions become identical, and the resulting configuration has layers extending vertically between the substrates in the "bookshelf" geometry. As $\theta_{0}$ increases from zero, the boundary conditions impose a bend deformation on the smectic. This deformation can be accommodated in several ways: by distributing the deformation over the vertical direction [Fig. 1(b)]; by localizing the bend to a region in the center with the layers flat and tilted in opposite directions in the top and bottom of the domain [Fig. 1(c)]; or by introducing edge disclinations to relieve the cost of elastic deformation [Figs. 1(d) and 1(e)].

Hence, the equilibrium structure as a function of $\theta_{0}$ is determined by an energetic competition between the cost of bending and the cost of introducing disclinations. Using a technique called deflation [73], we can compute a bifurcation diagram for this scenario and quantitatively determine

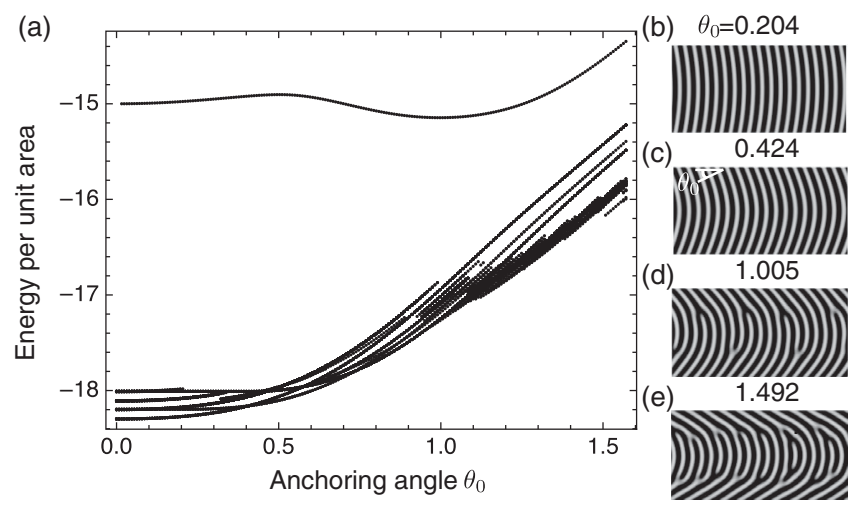

FIG. 1. Applying a bend deformation to a smectic liquid crystal. (a) Bifurcation diagram. (b)-(e) Stable stationary solutions for different values of $\theta_{0}$. The visualization displays the density variation $\delta \rho$. which of these solutions is the ground state as a function of $\theta_{0}$ [Fig. 1(a)]. Readers may refer to the Supplemental Material [60] for full details of the problem setup, an extended presentation of more stationary configurations computed in this scenario, and a video illustrating the lowest-energy solutions found as $\theta_{0}$ is varied (all of which are stable).

A more extreme scenario is where the preferred alignment axes at each surface are perpendicular: One favors planar and the other vertical alignment. The experimentally observed configurations in this case are known as toroidal focal conic domains (TFCDs): The smectic layers adopt a configuration consisting of stacked interior sections of tori, with a central line defect extending between the two substrates. TFCDs may exist as isolated domains in a background of vertically oriented smectic layers, or they may self-assemble into a hexagonal lattice $[37,38,44,45]$. If one of the boundary conditions is perturbed, such as by introducing a small preferred tilt at either substrate, asymmetric FCDs may arise where the layers form from sections of Dupin cyclides [37,44].

Despite the centrality of TFCDs in the study of smectics, and for applications, prior numerical work has been limited to finding solutions using modifications of the nematic theory [43]. Therefore, we verify that FCDs are stationary solutions of our functional (3) and characterize their response to tilted boundary conditions. Specifically, we perturb the zenith angle $\theta_{c}$ between the director and the $z$ axis in the boundary configuration (see detailed descriptions in the Supplemental Material [60]). Displayed in Fig. 2 is a sequence of solutions as a function of $\theta_{c}$, the preferred tilt away from the vertical at the upper substrate. All solutions displayed are stable.

As can be seen in Fig. 2(c), we recover the cylindrically symmetric TFCD for $\theta_{c}=0$; as $\theta_{c}>0$, the solution (a)

(c)
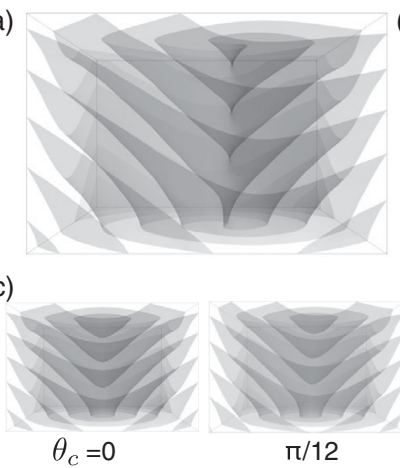

(b)
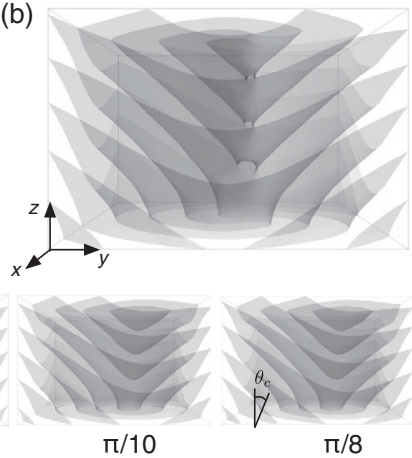

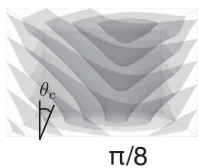

FIG. 2. Focal conic domains under tilted boundary conditions. Example solutions with (a) single- and (b) double-screw dislocation defects at $\theta_{c}=\pi / 12$. (c) Stable stationary solutions for different values of $\theta_{c}$. Here, zero isosurfaces of the density variation $\delta \rho$ are displayed to visualize the layer structure of the smectic. Among the three solutions shown for $\theta_{c}=\pi / 12$, the FCD solution possesses the lowest energy value, while the double-screw dislocation solution has the highest value. 


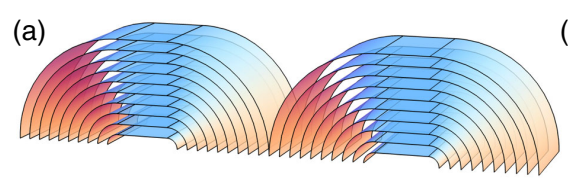

(d)

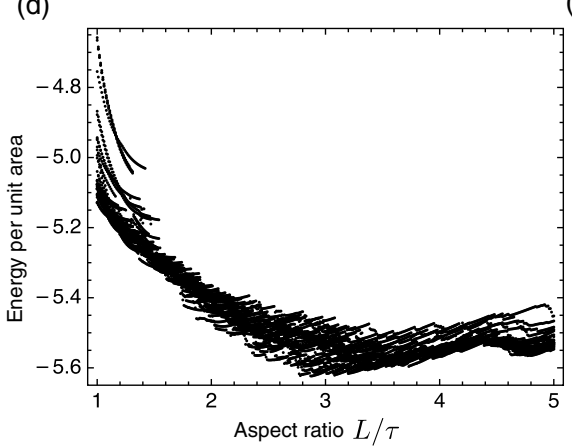

(b)

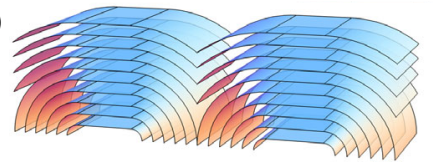

(e) $L / \tau=1.5$

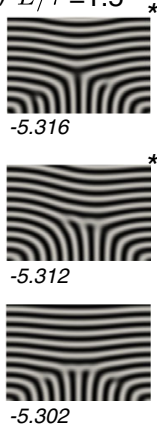

2.88 (c)
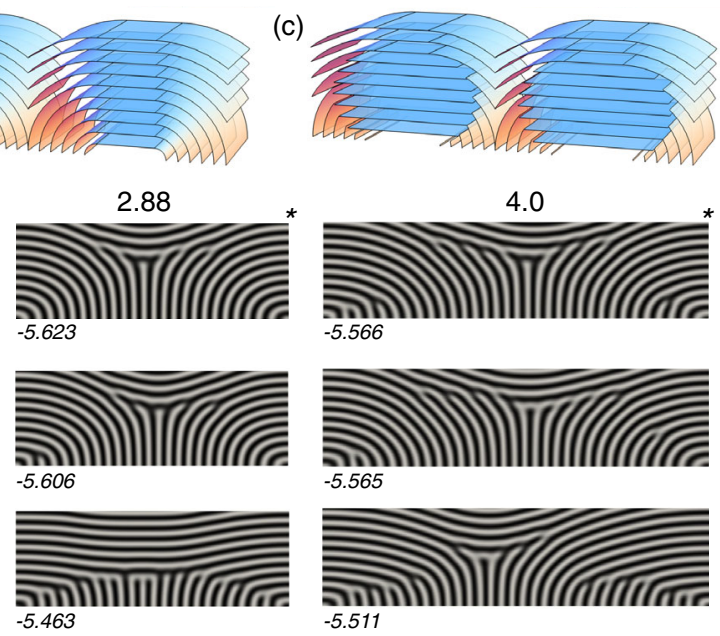

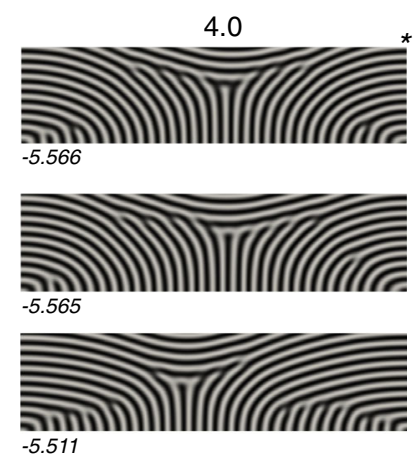

FIG. 3. Oily streaks. (a)-(c) Candidate structures proposed in Michel et al. [42] consistent with x-ray diffraction. (d) Bifurcation diagram of structures as a function of aspect ratio $L / \tau$. (e) Selected stationary states obtained at different aspect ratios $L / \tau$. The top row represents the lowest-energy solution found. For each solution, the value of the energy functional per unit area is displayed below it, with asterisks indicating stable profiles.

becomes asymmetric [Fig. 2(c)], and the central defect line becomes a hyperbola, as expected from geometry $[3,4,18]$. Three examples of the solution structures [including the single-screw dislocation defect presented in Fig. 2(b)] at $\theta_{c}=\pi / 12$ are shown in the Supplemental Material [60].

For sufficiently thin films, the elastic energy cost of two-dimensional curvature of the layers observed in the FCD solutions becomes prohibitive. Instead, the smectic adopts a configuration referred to as an "oily streak" texture $[21,41,43]$. The structure is periodic in one direction parallel to the substrate and spatially uniform in the other, tangential direction; the periodicity $L$ is experimentally found to increase linearly with the film thickness $\tau$ such that $L \approx L_{0}+4.5 \tau$ [42]. Addition of chiral dopants can be used to control the orientation of the streaks [41].

X-ray diffraction experiments for films $0.15 \mu \mathrm{m} \leq \tau \leq$ $0.35 \mu \mathrm{m}$ (47-110 layers) indicate that the smectic-layer normals are continuously and uniformly distributed in orientation, with a significant additional peak for smectic layers that are parallel to the plane of the substrate. An approximate layer structure proposed by Michel et al. [42], which is consistent with these data, comprises periodic units incorporating sections of cylinders joined to planes oriented parallel to the substrate [Fig. 3(a)]. However, this structure implies significant deformations of the free interface with singular points between units; while undulations of the smectic-air interface are observed by atomic force microscopy, the amplitude is only around $1 / 5$ of the film thickness once the finite size of the tip is accounted for. To address this issue, the same authors consider more complex structures incorporating curvature walls [21,43] [Fig. 3(b) that necessarily imply local dilation of the layers or local melting into the nematic phase along walls between units.

For even thinner films, $\tau \sim 70 \mathrm{~nm}$ (approx. 22 layers), $\mathrm{x}$-ray diffraction reveals an apparent excess of the planar region that cannot be explained by either structure discussed so far [43]; a possible structure that is consistent with the X-ray data is depicted in Fig. 3(c); it incorporates an approximately hemicylindrical rotating grain boundary (RGB) that partitions the cylindrical component from the planar component. Such a structure, with abruptly discontinuous layers, is energetically very costly and was envisioned in Ref. [43] as a mesoscopic approximation: At the nanoscopic level, the RGB might contain a network of dislocations to include the additional layers, or it might locally melt into the nematic phase along the RGB.

Hence, while these ansatz models are very helpful in that they provide an overall understanding of the structure and facilitate interpretation of the experimental data, they incorporate coarse-grained features such as the RGBs, and moreover, they are not calculated stationary states of an appropriate free energy functional. Understanding the detailed structure of the oily streaks therefore remains an important open problem.

We again use the deflation technique to explore the stationary states of Eq. (3) on a rectangular domain of fixed vertical dimension and varying aspect ratio $L / \tau$. For simplicity, we do not allow for variation of the free surface, which will be an area of future work, but we instead impose weak anchoring conditions. As with the other numerical experiments, full details of the boundary conditions, solvers, and choices of parameters are documented in the Supplemental Material [60]. Furthermore, a video showing the lowest-energy configurations as the aspect ratio $L / \tau$ varies is also included; all of these configurations are stable.

A partially enumerated energy landscape is displayed in Fig. 3(d), showing an extremely dense thicket of solutions but qualitatively supporting earlier work in that an overall minimizer occurs at an aspect ratio of around 3, which is similar to experimental values even with no parameter 
tuning. Close examination of the energy landscape, together with the corresponding solution set, shows many small discontinuous jumps that result from delicate commensurability effects, whereby certain domain sizes are compatible with a given periodicity of the layers, as well as from variations in the number of defects and their detailed placement. Similar effects have been observed when other periodic liquid crystals such as cholesterics are confined in domains that promote geometric frustration [74].

The solution set we obtain contains examples reminiscent of previously proposed structures [Fig. 3(e)]. The minimum energy states found at different aspect ratios contain cylindrical sections mediated by a defect-filled region reminiscent of the mesoscopic rotating grain boundaries. Other solutions, displayed in the lowest row of Fig. 3(e), are quite different from those heretofore proposed, where regions of relatively vertically oriented layers sit atop cylindrical regions interspersed with defects. Each of these incorporates a greater proportion of vertical layers relative to the hemicylindrical-planar ansatz of Figs. 3(a) and 3(b), and may provide alternative structures for oily streaks in ultrathin films. In future work, the boundary conditions at the top interface should be carefully reconsidered, including the incorporation of a free interface.

In conclusion, we have formulated a free energy functional for smectics that is amenable to finite element simulation, and we have applied it to scenarios involving boundary conditions that are incompatible with uniform smectic order; our new model successfully reproduces, even without careful tuning of parameters, a number of experimentally observed and theoretically expected phenomena; in addition, it produces new candidate structures for thin smectic films that are explicitly stationary states of an energy functional. We also demonstrate how to overcome a less obvious difficulty with numerical studies of smectics and layered media, in general: The solution landscapes are extremely dense because of the presence of defects. The combination of our model and the deflation technique enables detailed exploration of this landscape, allowing us to isolate both the ground state and low-lying excited states that may be observed in physical systems.

The work of J. X. is supported by the National University of Defense Technology and the EPSRC Centre for Doctoral Training in Partial Differential Equations (Grant No. EP/ L015811/1). The work of S. M. was partially supported by a NSERC Discovery Grant. The work of P.E. F. was supported by EPSRC Grants No. EP/R029423/1 and No. EP/ V001493/1. The work of T. J. A. was partially supported by NSF Grants No. DMR-1654283 and No. OAC-2003820.

*Corresponding author. timothy.atherton@tufts.edu

† Corresponding author. patrick.farrell@maths.ox.ac.uk
[1] P. G. de Gennes, Solid State Commun. 10, 753 (1972).

[2] C. S. Rosenblatt, R. Pindak, N. A. Clark, and R. B. Meyer, J. Phys. France 38, 1105 (1977).

[3] M. Kléman and O. D. Lavrentovich, Phys. Rev. E 61, 1574 (2000).

[4] M. Kléman and O. D. Lavrentovich, Liq. Cryst. 36, 1085 (2009).

[5] I. W. Stewart, Liq. Cryst. 15, 859 (1993).

[6] G. Friedel and F. Grandjean, Bull. Soc. Fr. Mineral. 33, 409 (1910).

[7] J. P. Bramble, S. D. Evans, J. R. Henderson, T. J. Atherton, and N. J. Smith, Liq. Cryst. 34, 1137 (2007).

[8] W. Guo, S. Herminghaus, and C. Bahr, Langmuir 24, 8174 (2008).

[9] E. Smela and L. J. Martinez-Miranda, J. Appl. Phys. 73, 3299 (1993).

[10] M. C. Choi, T. Pfohl, Z. Wen, Y. Li, M. W. Kim, J. N. Israelachvili, and C. R. Safinya, Proc. Natl. Acad. Sci. U.S.A. 101, 17340 (2004).

[11] T. Ohzono, Y. Takenaka, and J.-I. Fukuda, Soft Matter 8, 6438 (2012).

[12] R. S. Preusse, E. R. George, S. A. Aghvami, T. M. Otchy, and M. A. Gharbi, Soft Matter 16, 8352 (2020).

[13] A. Honglawan, D. A. Beller, M. Cavallaro, R. D. Kamien, K. J. Stebe, and S. Yang, Proc. Natl. Acad. Sci. U.S.A. 110, 34 (2013).

[14] Y. Xia, A. A. DeBenedictis, D. S. Kim, S. Chen, S.-U. Kim, D. J. Cleaver, T. J. Atherton, and S. Yang, Nat. Commun. 10, 5104 (2019).

[15] T. Lopez-Leon and A. Fernandez-Nieves, Colloid Polym. Sci. 289, 345 (2011).

[16] M. Urbanski, C. G. Reyes, J. Noh, A. Sharma, Y. Geng, V. S. R. Jampani, and J. P. F. Lagerwall, J. Phys. Condens. Matter 29, 133003 (2017).

[17] X. Xing, H. Shin, M. J. Bowick, Z. Yao, L. Jia, and M.-H. Li, Proc. Natl. Acad. Sci. U.S.A. 109, 5202 (2012).

[18] C. Blanc and M. Kléman, Eur. Phys. J. E 4, 241 (2001).

[19] M. A. Gharbi, I. B. Liu, Y. Luo, F. Serra, N. D. Bade, H.-N. Kim, Y. Xia, R. D. Kamien, S. Yang, and K. J. Stebe, Langmuir 31, 11135 (2015).

[20] F. Serra, M. A. Gharbi, Y. Luo, I. B. Liu, N. D. Bade, R. D. Kamien, S. Yang, and K. J. Stebe, Adv. Opt. Mater. 3, 1287 (2015).

[21] J.-P. Michel, E. Lacaze, M. Alba, M. de Boissieu, M. Gailhanou, and M. Goldmann, Phys. Rev. E 70, 011709 (2004).

[22] Y. H. Kim, D. K. Yoon, H. S. Jeong, O. D. Lavrentovich, and H. Jung, Adv. Funct. Mater. 21, 610 (2011).

[23] D. P. Singh, R. Visvanathan, A. E. Duncan, B. Duponchel, Y. Boussoualem, S. Kumar, N. A. Clark, J.-F. Blach, R. Douali, and A. Daoudi, Liq. Cryst. 46, 1 (2019).

[24] I. Gryn, E. Lacaze, L. Carbone, M. Giocondo, and B. Zappone, Adv. Funct. Mater. 26, 7122 (2016).

[25] S.-P. Do et al., Nano Lett. 20, 1598 (2020).

[26] A. Honglawan, D. S. Kim, D. A. Beller, D. K. Yoon, M. A. Gharbi, K. J. Stebe, R. D. Kamien, and S. Yang, Soft Matter 11, 7367 (2015).

[27] Y. Mai and A. Eisenberg, Chem. Soc. Rev. 41, 5969 (2012).

[28] H. Hu, M. Gopinadhan, and C. O. Osuji, Soft Matter 10, 3867 (2014). 
[29] M. Selmi, J.-C. Loudet, P. V. Dolganov, T. Othman, and P. Cluzeau, Soft Matter 13, 3649 (2017).

[30] L. S. Hirst, A. Ossowski, M. Fraser, J. Geng, J. V. Selinger, and R. L. B. Selinger, Proc. Natl. Acad. Sci. U.S.A. 110, 3242 (2013).

[31] M. A. Gharbi, D. A. Beller, N. Sharifi-Mood, R. Gupta, R. D. Kamien, S. Yang, and K. J. Stebe, Langmuir 34, 2006 (2018).

[32] G. P. Alexander, B. G.-g. Chen, E. A. Matsumoto, and R. D. Kamien, Phys. Rev. Lett. 104, 257802 (2010).

[33] D. A. Beller, M. A. Gharbi, A. Honglawan, K. J. Stebe, S. Yang, and R. D. Kamien, Phys. Rev. X 3, 041026 (2013).

[34] J. P. Sethna and M. Kléman, Phys. Rev. A 26, 3037 (1982).

[35] M. Kléman, J. Phys. France 38, 1511 (1977).

[36] D. B. Liarte, M. Bierbaum, M. Zhang, B. D. Leahy, I. Cohen, and J. P. Sethna, Phys. Rev. E 92, 062511 (2015).

[37] M.-J. Gim, D. A. Beller, and D. K. Yoon, Nat. Commun. 8, 15453 (2017).

[38] T. J. Atherton, R. Wang, and C. Rosenblatt, Phys. Rev. E 77, 061702 (2008).

[39] D. Coursault, J. Grand, B. Zappone, H. Ayeb, G. Lévi, N. Félidj, and E. Lacaze, Adv. Mater. 24, 1461 (2012).

[40] D. Coursault, B. Zappone, A. Coati, A. Boulaoued, L. Pelliser, D. Limagne, N. Boudet, B. H. Ibrahim, A. d. Martino, M. Alba, M. Goldmann, Y. Garreau, B. Gallas, and E. Lacaze, Soft Matter 12, 678 (2016).

[41] I. R. Nemitz, A. J. Ferris, E. Lacaze, and C. Rosenblatt, Soft Matter 12, 6662 (2016).

[42] E. Lacaze, J.-P. Michel, M. Alba, and M. Goldmann, Phys. Rev. E 76, 041702 (2007).

[43] J.-P. Michel, E. Lacaze, M. Goldmann, M. Gailhanou, M. deBoissieu, and M. Alba, Phys. Rev. Lett. 96, 027803 (2006).

[44] B. Zappone, C. Meyer, L. Bruno, and E. Lacaze, Soft Matter 8, 4318 (2012).

[45] P. E. Cladis and S. Torza, J. Appl. Phys. 46, 584 (1975).

[46] B. Zappone, A. E. Mamuk, I. Gryn, V. Arima, A. Zizzari, R. Bartolino, E. Lacaze, and R. Petschek, Proc. Natl. Acad. Sci. U.S.A. 117, 17643 (2020).

[47] L. J. Martinez-Miranda, Y. Shi, and S. Kumar, Mol. Cryst. Liq. Cryst. Sci. Technol., Sect. A 326, 41 (1999).

[48] K. May, K. Harth, T. Trittel, and R. Stannarius, Chem. Phys. Chem. 15, 1508 (2014).

[49] K. May, K. Harth, T. Trittel, and R. Stannarius, Europhys. Lett. 100, 16003 (2012).

[50] K. Harth, T. Trittel, K. May, and R. Stannarius, Soft Matter 15, 6769 (2019).

[51] M. Y. Pevnyi, J. V. Selinger, and T. J. Sluckin, Phys. Rev. E 90, 032507 (2014).

[52] J. M. Ball, Mol. Cryst. Liq. Cryst. 647, 1 (2017).

[53] J. P. Borthagaray, R. H. Nochetto, and S. W. Walker, Numer. Math. 145, 837 (2020).

[54] C. E. Williams and M. Kléman, J. Phys. Colloq. 36, C1-315 (1975).

[55] A. Linhananta and D. E. Sullivan, Phys. Rev. A 44, 8189 (1991).

[56] A. Poniewierski and T. J. Sluckin, Phys. Rev. A 43, 6837 (1991).
[57] J. M. Ball and S. J. Bedford, Mol. Cryst. Liq. Cryst. 612, 1 (2015).

[58] A. Majumdar and A. Zarnescu, Arch. Ration. Mech. Anal. 196, 227 (2010).

[59] S. C. Brenner, $C^{0}$ Interior Penalty Methods, in Frontiers in Numerical Analysis-Durham 2010. Lecture Notes in Computational Science and Engineering, Vol. 85, edited by J. Blowey and M. Jensen (Springer, Berlin, Heidelberg, 2011), https://doi.org/10.1007/978-3-642-23914-4_2.

[60] See Supplemental Material at http://link.aps.org/ supplemental/10.1103/PhysRevLett.126.177801 for a detailed description of the implementation used in this Letter, where to find the exact versions of the code used to generate our results, a video illustrating the lowest-energy solutions as $\theta_{0}$ is varied in the defect-free scenario, a video illustrating the lowest-energy solutions as the aspect ratio $L / \tau$ is varied in the oily-streaks scenario, and one more video illustrating the structures of three different types of solutions (FCD, single-screw, and double-screw dislocations) found at $\theta_{c}=$ $\pi / 12$ as described in the example of Fig. 2, which includes Refs. [61-72].

[61] J. Xia, Smectic-A numerics (2021), https://doi.org/10.5281/ zenodo.4607849

[62] Software used in "Structural Transitions in Geometrically Frustrated Smectics" (2021), https://doi.org/10.5281/ zenodo.4441123.

[63] A. Dener, A. Denchfield, H. Suh, T. Munson, J. Sarich, S. Wild, S. Benson, and L. C. McInnes, https://www.mcs.anl .gov/petsc/petsc-current/docs/tao_manual.pdf.

[64] V. Hernandez, J. E. Roman, and V. Vidal, ACM Trans. Math. Softw. 31, 351 (2005).

[65] G. W. Stewart, SIAM J. Matrix Anal. Appl. 23, 601 (2001).

[66] J. Nocedal and S.J. Wright, Numerical Optimization (Springer, Berlin, 1999), ISBN 0387987932.

[67] R. Seydel, Practical Bifurcation and Stability Analysis, Interdisciplinary Applied Mathematics Vol. 5 (Springer, Berlin, 2010), ISBN 978-1-4419-1740-9.

[68] S. Balay, S. Abhyankar, M. F. Adams, J. Brown, P. Brune, K. Buschelman, L. Dalcin, V. Eijkhout, W. D. Gropp, D. Kaushik, M. G. Knepley, L. C. McInnes, K. Rupp, B. F. Smith, and H. Zhang, https://www.mcs.anl.gov/petsc/petsccurrent/docs/manual.pdf.

[69] F. Rathgeber,D. A. Ham,L. Mitchell, M. Lange,F. Luporini, A. T. T. Mcrae,G.-T. Bercea, G. R. Markall, and P. H. J. Kelly, ACM Trans. Math. Softw. 43, 1 (2016).

[70] P. R. Amestoy, I. Duff, and J.-Y. L'Excellent, Comput. Methods Appl. Mech. Eng. 184, 501 (2000).

[71] P. R. Brune, M. G. Knepley, B. F. Smith, and X. Tu, SIAM Rev. 57, 535 (2015).

[72] J. Ahrens, B. Geveci, and C. Law, Visualization Handbook, edited by C. D. Hansen and C. R. Johnson (ButterworthHeinemann, Burlington, 2005), pp. 717-731, ISBN 978-012-387582-2.

[73] P. E. Farrell, Á. Birkisson, and S. Funke, SIAM J. Sci. Comput. 37, A2026 (2015).

[74] D. B. Emerson, J. H. Adler, P. E. Farrell, S. P. MacLachlan, and T. J. Atherton, Liq. Cryst. 45, 341 (2018). 